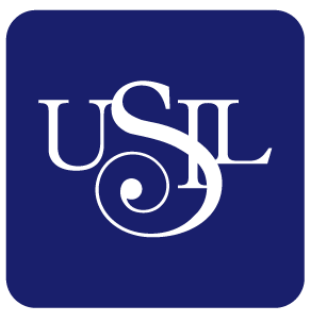

UNIVERSIDAD

SAN IGNACIO

DE LOYOLA

ESCUELA DE POSTGRADO

Maestría en Educación con mención en Docencia en Educación Superior

\title{
ESTRATEGIA DIDÁCTICA PARA EL DESARROLLO DEL PENSAMIENTO CRÍTICO EN LOS ESTUDIANTES DEL SEGUNDO CICLO DE UNA UNIVERSIDAD PRIVADA DE LIMA
}

Tesis para optar el grado de Maestro en Educación con mención en Docencia en Educación Superior

\section{SHERIDAN ZOZERI MEDINA CABRERA}

Asesor:

Félix Fernando Goñi Cruz

Lima - Perú

2019 


\section{Dedicatoria}

A mi familia, porque la educación es un privilegio al que sin ellos no hubiese podido acceder. 


\section{Agradecimientos}

A Paremos el Acoso Callejero, organización cuyo activismo despertó y motivó en mí la vocación docente y el deseo de especializarme para ser parte de la trasformación de esta sociedad a través de la educación.

Esta investigación no hubiese sido posible de realizar sin el apoyo constante de mis maestros de la Maestría de Educación con mención en Docencia Superior de la Universidad San Ignacio de Loyola. A ellos, mi agradecimiento, pues no solo se encargaron de conducir los cursos necesarios para el desarrollo de nuestras tesis, sino que, además, nos motivaron y sostuvieron a lo largo de todo el proceso.

Por otro lado, agradezco de manera especial a mi asesor de tesis, el doctor Fernando Goñi, quien, con paciencia y disciplina, motivó, dirigió y encausó en más de una ocasión esta investigación. No solo fue un asesor académico, fue además un mentor de nuestra vocación docente. A él toda mi admiración y gratitud.

Finalmente, quisiera agradecer a mi compañera, amiga y hoy colega Lesley Regalado, por enseñarme la trascendencia y pasión de la vocación docente, por su cariño y apoyo constante y por ser mi soporte emocional durante el programa de la maestría y de esta investigación. 


\section{Índice}

$\begin{array}{ll}\text { Dedicatoria } & \text { ii }\end{array}$

$\begin{array}{ll}\text { Agradecimientos } & \text { iii }\end{array}$

Índice $\quad$ iv

Resumen $\quad$ ix

Abstract $-x$

$\begin{array}{ll}\text { Introducción } & 11\end{array}$

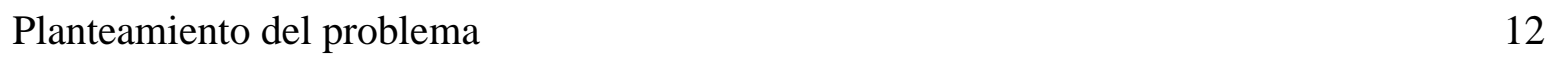

Formulación del problema 16

$\begin{array}{ll}\text { Preguntas científicas } & 16\end{array}$

$\begin{array}{ll}\text { Objetivos de investigación } & 17\end{array}$

$\begin{array}{ll}\text { Categorías y subcategorías apriorísticas } & 17\end{array}$

$\begin{array}{ll}\text { Justificación } & 18\end{array}$

Metodología de la investigación $\quad 19$

$\begin{array}{ll}\text { Tipo y diseño de investigación } & 20\end{array}$

$\begin{array}{ll}\text { Métodos de la investigación. } & 23\end{array}$

Técnicas e instrumentos para el trabajo de campo 26

$\begin{array}{ll}\text { Capítulo I } & 29\end{array}$

$\begin{array}{ll}\text { Marco teórico } & 29\end{array}$

Fundamentación teórica de la investigación $\quad 29$

Antecedentes de la investigación. $\quad 29$

$\begin{array}{ll}\text { Antecedentes internacionales. } & 29\end{array}$

Antecedentes nacionales. $\quad 32$ 
Fundamentos teóricos, epistemológicos y psicopedagógicos del desarrollo del pensamiento

crítico

Enfoques teóricos del aprendizaje

Aprendizaje constructivista

Aprendizaje significativo

Aprendizaje dialógico

Pensamiento crítico

El pensamiento crítico como competencia

Aproximaciones teóricas sobre la estrategia didáctica

Estrategia

Didáctica

Estrategia didáctica

Subcategorías de la estrategia didáctica según Perdomo

Capítulo II

Diagnóstico o trabajo de campo

Análisis, interpretación, triangulación y discusión de los resultados: Categorías emergentes.

Conclusiones aproximativas de los análisis realizados en esta parte de la investigación

Capítulo III

Estrategia Didáctica Dialógica para desarrollar Pensamiento Crítico (MEDD) 86

$\begin{array}{lc}\text { Fundamentación científica de la propuesta } & 88\end{array}$

$\begin{array}{ll}\text { Diseño gráfico funcional de la propuesta } & 105\end{array}$

Desarrollo o implementación de la propuesta 106

Validación de la propuesta 
Resultado de la valoración de los especialistas y conclusiones $\quad 131$

$\begin{array}{ll}\text { Conclusiones } & 133\end{array}$

$\begin{array}{ll}\text { Recomendaciones } & 135\end{array}$

$\begin{array}{ll}\text { Referencias } & 137\end{array}$

$\begin{array}{ll}\text { Anexos } & 145\end{array}$ 


\section{Índice de tablas}

Tabla 1. Resultados de la validación de instrumentos por juicio expertos

Tabla 2. Dimensiones del pensamiento crítico según Furedy y Furedy

Tabla 3. Subcategorías del pensamiento crítico según Faccione

Tabla 4. Subcategorías de la estrategia didáctica según Perdomo

Tabla 5. Tabla de competencias y capacidades

103

Tabla 6. Etapas de la propuesta

106

Tabla 7. Propuesta de talleres MEDD

Tabla 8. Tabla de Especialistas de la Validación.

Tabla 9. Tabla Validez interna por juicio de expertos

Tabla 10. Tabla de Validez externa por juicio de expertos

Tabla 11. Tabla de Escala de Valoración

Tabla 12. Tabla de Valoración interna y externa por criterio de jueces 


\section{Índice de figuras}

Figura 1. Categorías apriorísticas y emergentes.

68

Figura 2. Valoración interna y externa por criterio de jueces

133 


\section{Resumen}

La investigación propone el diseño de una estrategia didáctica para mejorar el desarrollo del pensamiento crítico en los estudiantes del segundo ciclo del curso de Comprensión y Producción de Lenguaje II de una universidad privada de Lima. Esta tesis se enmarca dentro del paradigma sociocrítico e interpretativo, de enfoque cualitativo y del tipo de investigación aplicada educacional. Respecto a la muestra, esta es intencionada de tres docentes y 25 estudiantes. Las técnicas que se emplearon fueron encuesta y entrevista. Los instrumentos utilizados fueron cuestionario, prueba pedagógica y guía de entrevista. El diagnóstico evidencia que los docentes trabajan con modelos operativos, administrativos o estrictamente didácticos que no están integrados en procesos pedagógicos dinámicos y creativos. Por otro lado, la sistematización teórica de las categorías apriorísticas se realizó basada en Faccione (1990), Bierman y Assali (1996), Mendoza (2015), Furedy y Furedy (1985), Gómez, 2015, Carrasco, 2016, y Gomero, 2009. El resultado es el diseño de una estrategia didáctica denominada Modelo de Estrategia Didáctica Dialógica (MEDD). La propuesta presenta en detalle las etapas, actividades e indicaciones para la práctica reguladora del docente en la implementación de la misma. Por todo lo anterior mencionado, concluimos que el estudio posee una característica formativa sólida, puesto que brinda una propuesta de solución al problema planteado a través de diversas herramientas pedagógicas.

Palabras clave: Investigación cualitativa, estrategia didáctica, pensamiento crítico, didáctica dialógica y práctica reguladora. 


\begin{abstract}
The research proposes the design of a didactic strategy to improve the development of critical thinking in students in the second cycle of the Language Comprehension and Production II course of a private university in Lima. This thesis is framed within the sociocritical and interpretive paradigm, with a qualitative approach and the type of applied educational research. Regarding the sample, it is intended by three teachers and 25 students. The techniques used were survey and interview. The instruments used were questionnaire, pedagogical test and interview guide. The diagnosis shows that teachers work with operational, administrative or strictly didactic models that are not integrated into dynamic and creative pedagogical processes. On the other hand, the theoretical systematization of the a priori categories was based on Faccione (1990), Bierman and Assali (1996), Mendoza (2015), Furedy and Furedy (1985), Gómez, 2015, Carrasco, 2016, and Gomero, 2009. The result is the design of a didactic strategy called the Dialogic Didactic Strategy Model (MEDD). The proposal presents in detail the stages, activities and indications for the teacher's regulatory practice in its implementation. For all the above mentioned, we conclude that the study has a solid formative characteristic, since it provides a proposal for a solution to the problem posed through various pedagogical tools.
\end{abstract}

Keywords: Qualitative research, didactic strategy, critical thinking, dialogical didactics and regulatory practice. 